\title{
Quantitative estimation of clay minerals in airborne hyperspectral data using a calibration field
}

\author{
Holger Eichstaedt, ${ }^{a} *$ Tugsbuyan Tsedenbaljir, ${ }^{b}$ Rene Kahnt, ${ }^{c}$ \\ Michael Denk, ${ }^{\mathrm{d}}$ Yaron Ogen ${ }^{\mathrm{d}}$ Cornelia Glaesser, ${ }^{\mathrm{d}}$ Ralf Loeser, ${ }^{\mathrm{c}}$ \\ Rudolf Suppes, ${ }^{\text {e }}$ Undrakhtamir Alyeksandr, ${ }^{\text {b }}$ Tsedendamba Oyunbuyan, \\ and Joseph Michalski ${ }^{f}$ \\ a'Dimap-Spectral GmbH, Freiberg, Germany \\ ${ }^{b}$ Erdenet Mining Corporation (State Owned Enterprise)—Friendship Square, \\ Bayan-Undursoum, Mongolia \\ ${ }^{\mathrm{c}}$ G.E.O.S. Ingenieurgesellschaft mbH, Halsbrücke, Germany \\ ${ }^{\mathrm{d}}$ Martin-Luther-Universität Halle-Wittenberg, Institute of Geosciences and Geography, \\ Department of Remote Sensing and Cartography, Halle, Germany \\ ${ }^{e}$ RWTH Aachen University, Institute of Mineral Resources Engineering, Aachen, Germany \\ ${ }^{\text {f } U n i v e r s i t y ~ o f ~ H o n g ~ K o n g, ~ D i v i s i o n ~ o f ~ E a r t h ~ a n d ~ P l a n e t a r y ~ S c i e n c e, ~ H o n g ~ K o n g, ~ C h i n a ~}$
}

\begin{abstract}
We evaluated the opportunities and performance of a new type of systematic pixel sharp calibration site for airborne hyperspectral mineral mapping in the environment of an operational copper deposit in Mongolia. The calibration site was designed to be used for estimation of sensitivity and quantification of key minerals in individual pixels in specific geological scenarios. The layout of the calibration site was done with two different copper-containing rock samples, a low copper-containing rock material from the mine, tailing material from the mine, and calibration materials with well-defined known spectral features. The scaled coverage of the sample materials was designed to develop statistical approaches to quantify target minerals in airborne surveys on a pixel-based approach. The data collection included the description of the calibration materials with geochemical, $\mathrm{x}$-ray diffraction, and microscopic and electron raster microscopic methods. Using visible and near-infrared airborne sensors and shortwave infrared airborne sensors, data of the calibration site were collected with multiple repeats from six altitudes. After rectification and atmospheric correction of pixels, sharp measurements of absorption features of clay minerals at 1400,1900, and $2200 \mathrm{~nm}$ were performed and statistically analyzed. Correlations between coverage and absorption features especially around $2200 \mathrm{~nm}$ are shown, and influences of flight altitude on sensitivity of the detection and the stability of the measurements are investigated. The results of the calibration field are used for the quantitative estimations of clay minerals in an exploration area near the mine site. The results are also shining new light on methodologies for ground truthing in hyperspectral surveys. () The Authors. Published by SPIE under a Creative Commons Attribution 4.0 Unported License. Distribution or reproduction of this work in whole or in part requires full attribution of the original publication, including its DOI. [DOI: 10 .1117/1.JRS.14.034524]
\end{abstract}

Keywords: airborne mineral exploration; visible and near-infrared/shortwave infrared hyperspectral; calibration; copper; clay.

Paper 200207 received Apr. 8, 2020; accepted for publication Sep. 4, 2020; published online Sep. 28, 2020.

\section{Introduction and Theoretical Background}

Hyperspectral infrared remote sensing has become an extremely useful and increasingly available tool for mineral exploration and geoscience in general over the last 40 years. ${ }^{1} \mathrm{~A}$ major goal of hyperspectral remote sensing has been the quantification of mineral abundance, not only for mineral exploration and geologic mapping on this planet but also for mineral mapping on other planets. Phyllosilicates, and specifically clay minerals, are a key mineral group of interest to

*Address all correspondence to Holger Eichstaedt, E-mail: he@dimap.com.au 
geologists as this group of finely crystalline phyllosilicates is known to trace hydrothermal and weathering processes relevant for understanding mineralization processes and geometries.

The most important spectral features for identification and mapping of clay minerals are the metal-OH vibrational overtones of the octahedral sheets (e.g., $\mathrm{AlOH}, \mathrm{MgOH}$, and $\mathrm{FeOH}$ located at wavelengths from $\sim 2160$ to $2350 \mathrm{~nm}$ ). ${ }^{2}$ To resolve these features, it is critical to have adequate spectral resolution. ${ }^{3}$ To relate the observed spectra to clay mineral abundances, it is also critical to understand the relationship between absorption strength and volume or weight abundance of the minerals in the target rock (and the abundance of the target rock in the observed pixel. ${ }^{4}$ Gilles et al. ${ }^{5}$ analyzed the abundance of different clay minerals in soils and compared $\mathrm{x}$-ray diffraction (XRD) measurements with estimations from spectroscopy using different methods of unmixing to estimate the potential of field spectroscopy for geotechnical applications.

The quantification of key minerals in airborne hyperspectral geological exploration over larger areas is complicated for various reasons, including heterogeneous solar exposition, the presence of vegetation, diverse geometries of rock materials, and the possibility that moisture variations in the top soils' information on quantity of key minerals are difficult to process. Attempts have been made to overcome these challenges with a combination of airborne, horizontal measurements of rock faces and outcrops combined with ground truthing. ${ }^{6,7}$ Field spectroscopy methods along with grab sampling and laboratory work provide calibration sources ${ }^{8}$ and can inform the development of expert systems for mineral mapping for specific regions. However, these approaches fall short in terms of quantitative information. ${ }^{9}$

While the usage of calibration fields is a standard approach for sensor calibrations in photogrammetrical applications, they are not common for hyperspectral sensors calibration. Markelin et al. ${ }^{10}$ proposed a classical radiance target test field to show the stability of reflectance measurements and test for spatial accuracy in hyperspectral sensors. They compare the results against the factory calibration of sensors from the manufacturer.

The following study sets the base for a research program inside the Adriana project to quantitatively measure the content of minerals in tailings and waste pile material as the basis for the development of a concept for reusing the material. In addition to this program for airborne missions with hyperspectral sensors, a wide variety of ground-based measurements, and an extensive sampling, and an analytical campaign, this publication investigates the opportunities for greenfield and brownfield exploration. A unique aspect of this program was a spectral calibration field, which includes realistic rock targets with relevant mineralogy. We aim to provide a new way to calibrate the data of airborne missions in mineral mapping. The main questions to be answered in this work are as follows.

- Is there a linear relationship between percent coverage and intensity of the spectral signal for the calibration materials and is that usable for quantitative estimation of target minerals?

- How do the results of the experiment relate to pixel size, geometry of the target materials, and set up of the calibration field?

- Using clay minerals as the proxy, what is the sensitivity of the hyperspectral measurement to mineral abundance?

- Is it possible to improve spectral investigations using calibration fields and how can the set up be improved?

\section{Material and Methods}

In August/September 2019, a multispectral airborne mission took place in Erdenet, Mongolia. The main focus was to perform a quantitative estimation of the mineral contents in the calibration field using hyperspectral data. Target rock samples were selected from a mine positioned in a prehistoric known copper deposit in the Central Asian Orogenic Belt-Selenge Intrusive Complex. Igneous rocks of the formation are calcite-alkaline, I-type, magnetite series, with medium to high $\mathrm{K}$ and enriched in low intensity linac (LIL). ${ }^{11}$ Key minerals of the deposit are bornite, chalcopyrite, galena, molybdenite, pyrite, sericite, sphalerite, and tennantite associated in rock formations of porphyries dominated by granodiorite, andesite, diorite and granites, as well as breccias. ${ }^{12}$ 
The experiment consisted of four main elements: (1) set up of the physical calibration field and geological targets, (2) carrying out the airborne flight measurements, (3) field spectral measurements carried out during flight, and (4) laboratory spectral measurements carried out later on the geological target materials. Each of these elements is described in detail below.

\subsection{Setup of the Calibration Field}

The calibration field was set on a concrete basketball court. The court was divided into squares of $4 \times 4 \mathrm{~m}^{2}$ in which the rock samples and calibration nets were placed. Figure 1 shows an aerial image of the calibration site and the description of the individual tiles with the final measured coverages of the different materials. The main goal of this investigation was to understand the relation of the distribution of the materials on their spectral signal considering the different pixel sizes as a result of different flight elevations. For this reason, the three different ore materials and reference materials were distributed in 48 tiles. The ore material was laid out in two coverage types-even over the whole tile in different abundances and center-concentrated in the middle of the tile, the final coverage percentage was measured based on the $2.5-\mathrm{cm}$ resolution orthophoto from the airborne mission as the number of pixels covered by the material, shadows, and remaining concrete are visible. In addition to the tiles that contained individual materials, other tiles that contained mixtures of sulphidic and oxidic rock materials were laid out in different concentrations and coverage types as well. Finally, the test field included empty tiles to represent bare concrete to describe the background and also by tiles covered with black net. The nets were black synthetic material similar to the one used by Brook and Ben-Dor ${ }^{13}$ for the supervised vicarious calibration of hyperspectral remote sensing data. Although this correction method was not applied in this research, different coverages of the black nets were used to investigate the statistical set up of the coverage. The test field set up of the tiles was optimized to allow the best separation of the tiles from each other in the hyperspectral data cubes. The spatial accuracy of the setup was verified with a real-time kinematic of Global Navigation Satellite Systems (GNSS).

\subsection{Description of the Geological Target Materials}

For the experiment, three typical rock types were provided by the mine operator, including a sulphide ore ( $\mathrm{Cu} 0.54 \%$ and Mo $0.0015 \%)$ (labeled M1 in this study), an ore of $34 \%$ oxidation (M2), and an oxidic waste ore with $\mathrm{Cu} 0.09 \%$ (M3). Additionally, tailing material with a grain size of $45 \mu \mathrm{m}$, based on the milling for the processing plant, and sand were laid out as targets.

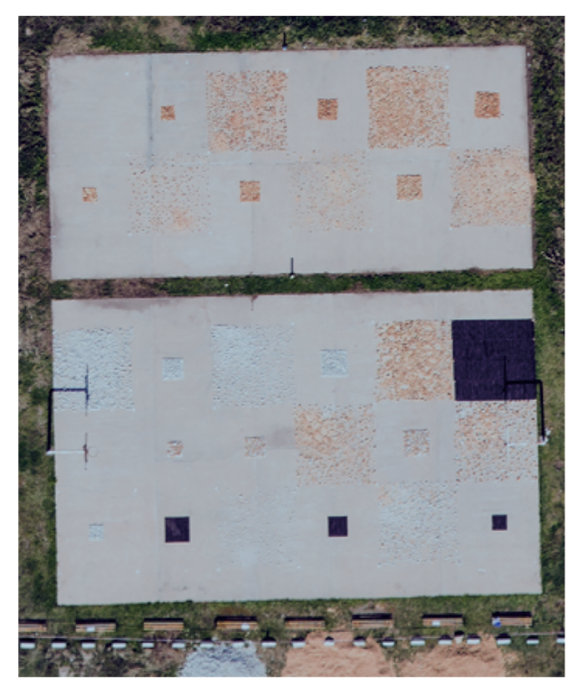

(a)

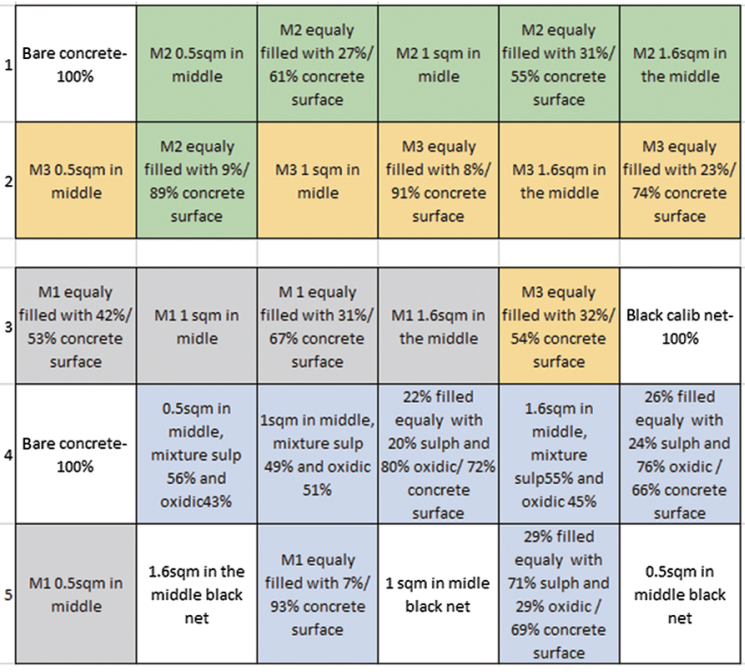

(b)

Fig. 1 (a) Main part of the calibration field-image Hasselblad H5DC 100-mm lens. (b) Short description of the individual tiles. 
These geological target materials were organized into closed and broken layers with rock samples of 5- to $15-\mathrm{cm}$ diameters.

Following are the description of the three samples following different analysis methods. In detail, the samples can be described as follows and mineral groups are given in Table 1.

Based on energy dispersive spectroscopy, material M1 is the sulphidic sample material containing abundant biotite overprinted by chlorite. The rock comprises $40 \%$ to $45 \%$ plagioclase, $25 \%$ to $30 \%$ quartz, $3 \%$ biotite, $5 \% \mathrm{k}$-spar, $5 \%$ sericite, $7 \%$ chlorite and clay minerals with kfeldspar, quartz, plagioclase with grain sizes ranging from 0.4 to $1.9 \mathrm{~mm}$ and minor tabular biotite. The plagioclase is partially overprinted by sericite. K-spar is rimmed by spotty albite along grain boundaries. Quartz is characterized by isometric grains with irregular boundaries. Biotite is completely overprinted by chlorite and occurs as $0.5-$ to $0.9-\mathrm{mm}$ tabular grains.

Material M2 is an oxidized ore sample composed of $42 \%$ to $45 \%$ plagioclase, $25 \%$ to $30 \%$ quartz, $5 \%$ to $10 \%$ feldspar, $8 \%$ sericite and secondary quartzite, $5 \%$ rock material with muscovite overprinted the biotite, and the fractures are filled by secondary quartz. It consists of plagioclase, 0.4- to 2.8-mm quartz, minor interstitial feldspar, and sericite. Quartz grains are 0.2 to $1.4 \mathrm{~mm}$, irregular, and isometric. Accessory minerals are represented by sphene and 0.04- to 0.4-mm apatite. Secondary quartz occurs as fracture fill.

Material M3 is also oxidized, similar to protolith, but is barren of copper and has higher feldspar and overall $\mathrm{Al}$ content.

Analysis of the clay minerals and species of the different rock material based on hyperspectral data illustrates the different clay typologies of the samples. The hyperspectral longwave

Table 1 Mineralogical composition of the sample rock material in mass percent based on TESCAN scanning electron microscope.

\begin{tabular}{|c|c|c|c|}
\hline Mineral groups & M1 & M2 & M3 \\
\hline Albite & 42.9579 & 42.3202 & 41.0467 \\
\hline Quartz & 29.3699 & 36.1708 & 27.5298 \\
\hline K_Feldspar & 4.3381 & 7.6952 & 14.1173 \\
\hline Muscovite & 8.5319 & 7.5954 & 4.1201 \\
\hline Alumosilicate mixture & 4.9291 & 0.4499 & 7.3035 \\
\hline Kaolinite & 3.1516 & 1.3039 & 1.7931 \\
\hline Other minerals & 0.6286 & 2.2824 & 0.4749 \\
\hline Chlorite & 1.9927 & 0.0005 & 1.3072 \\
\hline Pyrite & 1.6609 & 0.3674 & 0.3128 \\
\hline Chalcopyrite & 1.1021 & 0.0206 & 0.0062 \\
\hline Iron_Oxide & 0.0179 & 0.3503 & 0.6798 \\
\hline Calcite/Dolomite & 0.0415 & 0.0104 & 0.0815 \\
\hline Apatite & 0.0594 & 0.0325 & 0.0108 \\
\hline Chalcocite & 0.0566 & 0.009 & 0.0007 \\
\hline Bornite & 0.0543 & 0.0031 & 0 \\
\hline Biotite & 0.0022 & 0.0277 & 0.0247 \\
\hline Covellite & 0.0297 & 0.0003 & 0.0039 \\
\hline Others and unclassified & 1.0756 & 1.3604 & 1.187 \\
\hline Total & 100 & 100 & 100 \\
\hline
\end{tabular}


Table 2 Results of the XRD on the sample materials M1, M2, and M3 for bulk samples and fractions smaller than $2 \mu \mathrm{m}$ (in percent).

\begin{tabular}{|c|c|c|c|c|c|c|}
\hline \multirow[b]{2}{*}{ Mineral } & \multicolumn{3}{|c|}{ Bulk sample } & \multicolumn{3}{|c|}{$<2-\mu \mathrm{m}$ size fraction } \\
\hline & M1 & M2 & M3 & M1 & M2 & M3 \\
\hline Albite (Feldspar) & - & 38.1 & 36.6 & - & 5.3 & 16.1 \\
\hline Orthoclase (Feldspar) & - & 12.0 & 13.3 & - & 46.0 & 3.3 \\
\hline Labradorite (Feldspar) & - & - & - & - & - & 14.1 \\
\hline Quartz & 60.0 & 29.1 & 36.2 & 54.5 & - & 34.1 \\
\hline Chlorite & - & 4.6 & - & - & 23.3 & - \\
\hline Pyrite & 10.5 & - & - & 6.9 & - & - \\
\hline Muscovite (mica) & 15.0 & - & - & 30.3 & - & - \\
\hline Zinnwaldite (mica) & 5.8 & 3.7 & 6.1 & - & - & - \\
\hline Clay minerals & - & 5.4 & - & - & 17.2 & - \\
\hline Strontiumthiosulphate & 8.7 & 5.4 & 5.7 & 8.3 & 8.3 & 13.4 \\
\hline Omeprazole & - & - & - & - & - & 13.8 \\
\hline
\end{tabular}

microscopy with $25-\mu \mathrm{m}$ resolution shows strong signals of saponite, smectite, nontronite, montmorillonite, and weaker signals of illite and kaolinite. In M1, montmorillonite is slightly more dominant than in M2 and M3. M2 has the highest values of nontronite, and in M3 the dominant clay minerals are saponite and smectite.

The core scan of the rock material with $0.3-\mathrm{mm}$ pixel resolution in the shortwave infrared (SWIR) bands shows significantly more clay signals (saponite, montmorillonite, and illite) in M2 and M3 than in sample M1. However, M1 was more abundant in nontronite than in the other samples.

Using XRD for bulk samples and size separation, a $<2-\mu \mathrm{m}$ fraction shows a more detailed picture of the three sample materials. The sulphidic material is rich in quartz and shows in bulk and fine component micas and pyrites. The copper-rich oxidic sample M2 shows high measurements of orthoclase and chlorite. Kaolinite and other clay minerals are only in M2 found in measurable abundances, while other clay minerals were under the set sensitivity of the XRD set up. M3 also shows on the floated samples with typical clay signals, but they are under the response level (Table 2).

\subsection{Airborne Mission}

The airborne data collection for the calibration field was performed with a Cessna 208B with a dual camera hole equipped with Lidar Riegl Q780, metric camera H5Dc 50 Megapixel with 100-mm fixed focus lens, a hyperspectral scanner Hyspex VNIR1600 (160 bands, 400 to $1000 \mathrm{~nm}$ ), and SWIR320me (256 bands 1000 to $2500 \mathrm{~nm}$ ) — both hyperspectral sensors were flown with a field-of-view (FOV) expander. All sensors were flown between 28th and 30th of August 2019. The flight direction was, in each case, south to north. The flight program included flights at six altitudes above ground with numerous repeats in the altitude to assure statistical suitable observations (Table 3). All data were flown within 80 min of solar noon.

The GNSS trajectory of the flight mission was processed against the 5-km far GNSSreference station of the Erdenet Mining Corporation (EMC). An absolute aircraft position was achieved within $2 \mathrm{~cm}$ in $X Y Z$. Further orthorectification of the image data for the calibration field was based on 2-cm accurate measured corners of the field and control points on and around the calibration field. Hyperspectral data cubes were rectified with PARGE airborne image rectification from RESE ${ }^{14}$ based on a general boresight and further improved with additional control 
Table 3 Summary of the flight set up with altitude above ground, repeat fly overs, and the spatial resolutions of the individual sensors.

\begin{tabular}{lcccc}
\hline \hline & & \multicolumn{3}{c}{ Nominal spatial resolution } \\
\cline { 2 - 5 } $\begin{array}{l}\text { Altitude above } \\
\text { ground level }(\mathrm{m})\end{array}$ & $\begin{array}{c}\text { Fly over } \\
\text { repeats }\end{array}$ & Imagery $(\mathrm{cm})$ & VNIR $(\mathrm{m})$ & SWIR $(\mathrm{m})$ \\
\hline 480 & 4 & 2.5 & 0.35 & 0.7 \\
650 & 7 & 3.6 & 0.5 & 1 \\
800 & 3 & 4.0 & 0.6 & 1.2 \\
950 & 3 & 4.8 & 0.75 & 1.5 \\
1300 & 3 & 6.5 & 1.0 & 2.0 \\
2000 & 4 & 10 & 1.5 & 3.0 \\
\hline \hline
\end{tabular}

points from the GNSS survey on the calibration field using gridded resampling to optimize between spectral and spatial quality of the orthorectified data. The required spatial pass accuracy to accept the solution was better than a 0.35-pixel size for each individual data cube. Atmospheric correction was performed using atmospheric and topographic correction ${ }^{15}$ with support of ground measured spectra of the black nets and the concrete. The atmospheric correction was based on an urban model due to the presence of industrial and city structures as major components of the mission area. The atmospheric correction was based on a fixed water vapor value based on average estimations for each flight level and regression of the water vapor bands.

The wider area of the EMC mine, tailing dam, and Erdenet town in four flight levels $(600,1000,1500$, and $2000 \mathrm{~m}$ above ground level) were also flown over with the same sensor set. Data were processed with similar settings as the calibration site and used for checking the results in a practical application.

\subsection{Ground Support Measurements}

During the flight campaign, spectral ground measurements of materials of the calibration site were performed using a Spectral Evolution SR-3500 spectroradiometer. The SR-3500 covers a wavelength range 0.35 to $2.5 \mu \mathrm{m}$ with a spectral resolution of $3 \mathrm{~nm}$ at $700 \mathrm{~nm}, 8 \mathrm{~nm}$ at $1500 \mathrm{~nm}$, and $6 \mathrm{~nm}$ at $2100 \mathrm{~nm}$. The measurements were conducted using a bare fiber optic with 25-deg FOV and an approximate measurement distance of $1 \mathrm{~m}$ to the ground. Depending on the filling of the tiles, 10 to 20 spectral measurements were conducted. The measured spectra were calibrated to absolute reflectance values using a Zenith Lite ${ }^{\mathrm{TM}}$ diffuse reflectance panel of $95 \%$ reflectivity. Spectral preprocessing encompassed the removal of wavelength regions heavily affected by noise due to atmospheric water vapor absorptions at 1.4 and $1.9 \mu \mathrm{m}$, averaging of all spectra per tile and smoothing the spectra by applying a Savitzky-Golay filter.

\subsection{Setup of Supporting Laboratory Experiments}

To support the modeling of the linear quantitative relationship between coverage and spectral features of clay minerals, a laboratory experiment similar to the calibration field was set up. This included four Petri dishes with different watery mixtures of concrete, hardened and dried in the muffler oven, and then set up with different sizes from 5 to $15 \mathrm{~mm}$ of circular set tailing material from the Erdenet mine with a suitable covering thickness (i.e., optically thick, $>100 \mu \mathrm{m}$ ). The tailing material was used. It can be expected to have similar clay features as the ore material and has a uniform grain size of $45 \mu \mathrm{m}$ due to processing of the ore. All samples were dried. The set up of the four dishes was completed with $5 \%$ and $40 \%$ spectralon reflectance panels. These panels were used for the correction of the measured data to reflectance values using an empirical line correction. The realization of additional coverage measurements was done via spectral binning of different sized circular areas around the circular tailing material. In total, 110 setups were 
measured, distributed over the different concrete settings. Absorption features were measured on continuum removed spectra in which absorption features present in the spectra are normalized against a convex hull covering the total spectral range.

\subsection{Measurements of Spectral Parameters}

Spectral data from the data cubes were analyzed using ENVI image analysis functionalities under visual controlled conditions, meaning unclear pixel locations over the calibration field were excluded from the measurements. For the imagery acquired at a flight altitude of $480 \mathrm{~m}$, spectra were collected and averaged within a $3 \times 3$ pixel window, and for the $650-\mathrm{m}$ altitude data, this average window was defined as a $2 \times 2$ pixel. Faulty bands were spectrally averaged with neighboring bands. Furthermore, all spectral measurements were attributed with material, coverage type, and flight altitude. For the analysis of individual spectral bands, spectra were normalized using the continuum removal procedure. ${ }^{1}$ In total, 351 valid spectra were measured over the different materials, altitudes, and repeats. For the description of the 2200-nm absorption band, the average of the continuum removed the value of the bands at 2100 and $2240 \mathrm{~nm}$, which was subtracted by the value at the band on $2192 \mathrm{~nm}$, while for the description of the 1400- and $1900-\mathrm{nm}$ bands only one reference band on the lower site (1370 and $1850 \mathrm{~nm}$ ) was used.

To separate the individual clay minerals for all samples, a spectral linear unmixing procedure was applied using the spectra from the United States Geological Survey (USGS) library and the concrete spectra from the field measurement. For the linear unmixing, water bands were excluded and unit sum constrains were not used under the assumption that in addition to the concrete and the clay minerals, further minerals were also part of the rock material laid out over the calibration tiles. For the analysis, only evenly laid out calibration tiles were used. Tiles with unclear visual allocation were excluded.

\section{Results}

\subsection{Linearity Checks on Calibration Targets in the Calibration Field}

As a first-order validation, 47 scenes with black net fields were used to verify the viability of the calibration field concept and the methodology of the measurement techniques for the spectra. The black net folded four times provides a reflectance below $5 \%$ over the full spectral bandwidth of the hyperspectral sensor used. ${ }^{13}$ An average of the reflectance of each tile with different coverages of black net over all bands of the SWIR range was calculated and the damping of the signal of the concrete based on the coverage percentage with black net was observed. Figure 2 shows the correlation between the coverage percentage of the pixel with black net and the averaged reflectance signal over all bands of the SWIR sensor. There is a good linearity between coverage

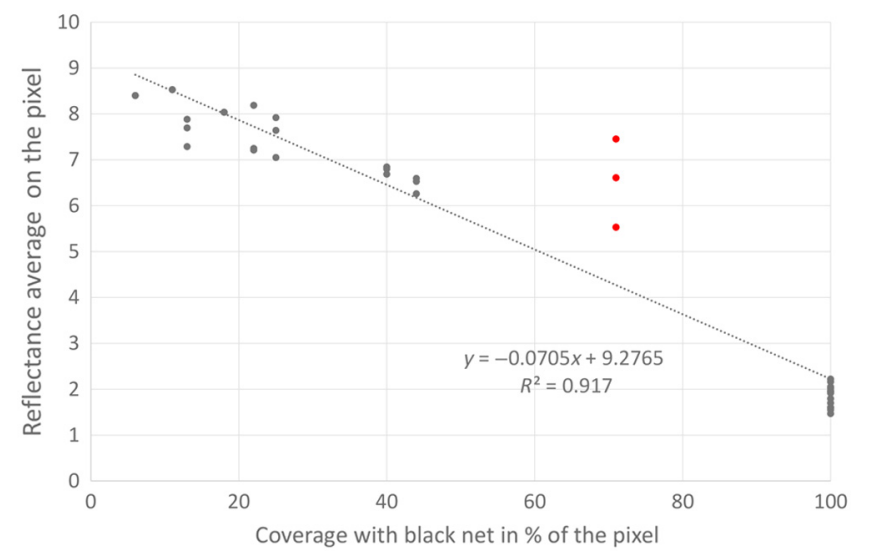

Fig. 2 Correlation between coverage percentage of the pixel with black net and the average reflectance value of the pixel in the SWIR bands-all flight altitudes included. 
and reflectance. The coverages below 50\% and at $100 \%$ show well-clumped points. However, the variability for the three measurements at $71 \%$ coverage is distinctively above the expected values. This can be explained with the spatial rectification of the data better than a 0.35 -pixel size. It shows that the spatial rectification error for a pixel with a partial coverage of more than $50 \%$ of the pixel size by a central target hampers the derivation of correct quantitative information, and therefore, is excluded from further analysis. Removing the measurements at $71 \%$ cover (red dots in Fig. 2) provides an $R^{2}$ of 0.98 . This supports the feasibility of the methodological approach of the calibration field.

\subsection{Usage of Clay Mineral Absorption Bands 1400, 1900, and $2200 \mathrm{~nm}$}

Clay minerals show strong absorption features in the SWIR at $1400 \mathrm{~nm}$ (caused by $\mathrm{OH}$ ), $1900 \mathrm{~nm}$ (caused by molecular water), and $2200 \mathrm{~nm}$ (caused by combined overtones of Al$\mathrm{OH}){ }^{2}$ Measurements of the depth of the absorption band and the area of the absorption were taken from continuum removed spectra. Figure 3 shows a sample spectrum of M2 with the dominant clay features.

The airborne imagery analyzed for evenly laid out material coverages of $8 \%, 23 \%$, and $31 \%$ of the tiles with materials M1 to M3, summarized over all flight altitudes recorded (total $n=54$ ), showed strong differences in the strength of the absorption bands at 1400, 1900, and $2200 \mathrm{~nm}$ and their variability as shown in Fig. 4. The absorption at $2200 \mathrm{~nm}$ showed the strongest relationship to the coverage of the pixel with the material M2. It also shows the smallest standard deviation and a clear increase of absorption signal with coverage. For the materials M1 and M3, similar behaviors could be found. For this reason, the further detailed analysis of the clay absorption bands is focused on the $2200 \mathrm{~nm}$. The poor correlations in 1400 and $1900 \mathrm{~nm}$ are due to overlays with strong water vapor bands and the difficulties in atmospheric correction (Fig. 4).

In a next step, even and centered laid out coverage tiles below 50\% were analyzed for the dependencies of the depth and area of the absorption band at $2200 \mathrm{~nm}$ to different parameters.

A relative absorption depth normalized to the coverage in percent was calculated to analyze the influence of the flying height on the depth of the absorption band. This relative depth was based on the assumption of linearity as shown above. The average relative absorption band depth shows no significant difference between the different flying heights. The error interval is increasing with the flying altitude, which might partly be due to the usage of the pixel-based averaging of the spectral measurements in the lower altitudes ( $480 \mathrm{~m}: 3 \times 3$ pixels, $650 \mathrm{~m}: 2 \times 2$ pixels). Figure 4 shows that, for the designed calibration field, the flying altitude is not of major importance and that using lower altitudes allows better statistics with reduced efforts for the set up of the calibration field, which is also supported by an analysis of variance. Comparing the three lower altitudes shows that up to $950 \mathrm{~m}$, similar behavior can be found for all three target materials, but there is an increase on the confidence interval of the width for the average with increasing altitude.

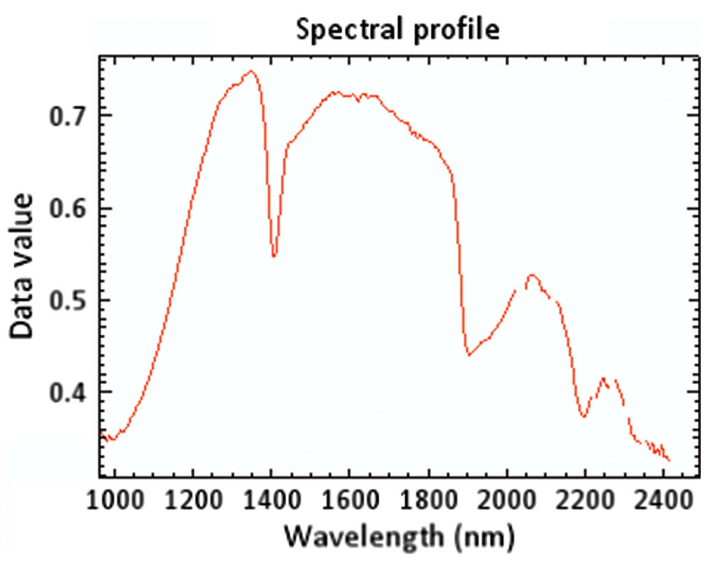

Fig. 3 Spectrum of material M2 based on grab sample scan with Hyspex SWIR with 0.3-mm resolution and corrected with spectrolons. 


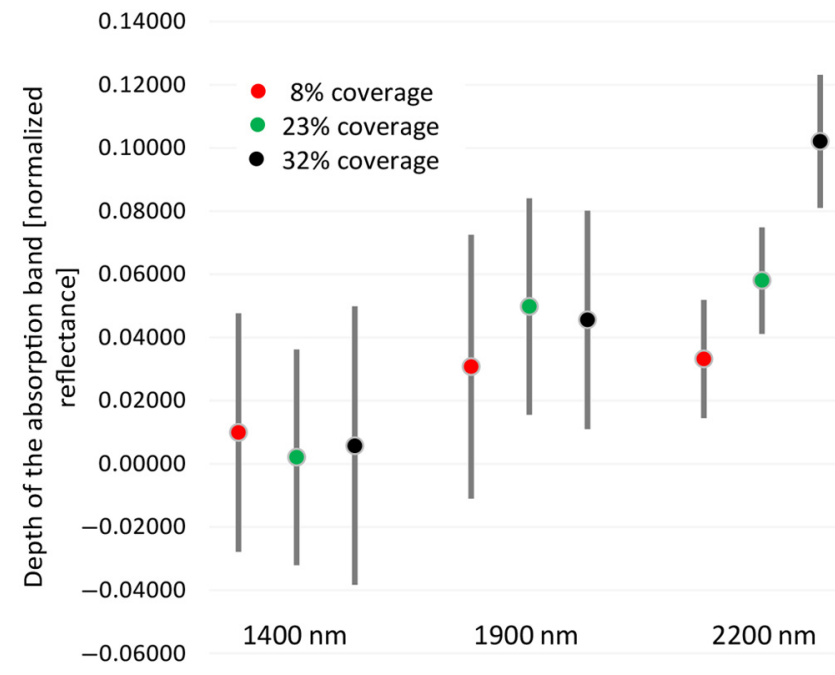

Fig. 4 Average and standard deviation for the depth of the three absorptions bands of clay minerals for rock type M2. $N$ per case and coverage is $18 \%$ of the tile.

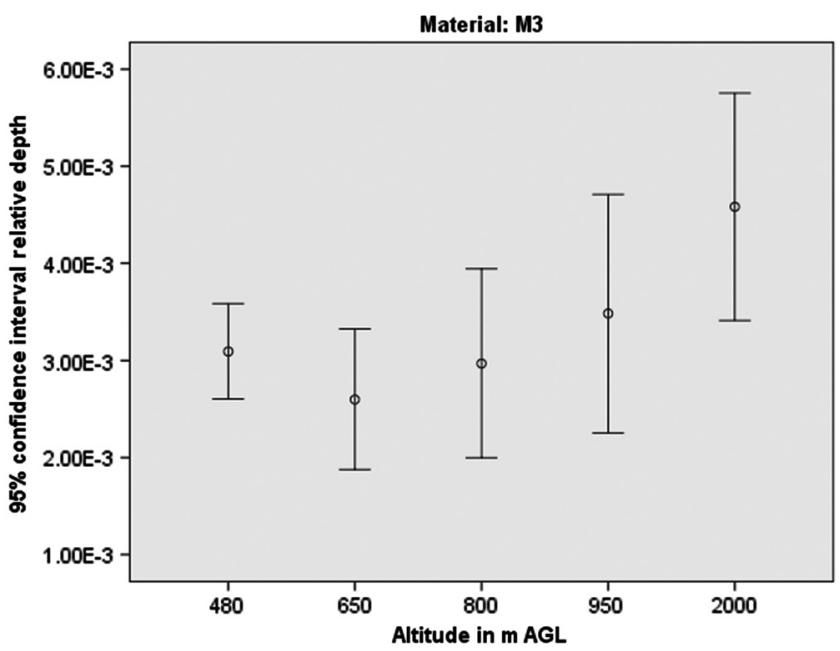

Fig. 5 Average and $95 \%$ confidence intervals of the relative depth of the 2200-nm absorption band plotted by the flying heights, an example for rock type M3 $(n=75)$.

Figure 5 shows the comparison of the relative absorption depths of the 2200-nm feature for the three materials between only center-filled and evenly filled tiles. While the averages are similar, the centered laid out samples have a higher error based on a smaller number of samples and the above-described complications of the spectral data on the pixels. This means again that evenly laid out calibration tiles with target rock samples are preferable. For estimating linearity of the coverage/absorption band solution, the centered tile design can be used to get supporting data points, especially in the lower coverage percentages, but the values measured have to be weighted lower.

Figure 6 shows the linearity of the tile coverage in relation to the absorption depth and the area for the three materials, respectively. The absorption depth shows similar tendencies for the oxidized materials M2 and M3, while material M1 shows a weaker increase with the coverage due to the lower clay contents. The linearity of the absorption band depth in evenly filled tiles is $R=0.70$ for M2 and $R=0.82$ for M3 higher than for M1 with $R=0.65$. For the target materials solely laid out in the center of the tiles, the correlation between coverage and absorption strength of the $2200 \mathrm{~nm}$ band was found to be weaker, which is most likely due to the aforementioned higher errors in this setting. The correlations observed for the individual scenarios per flying altitude and coverage type show better fits to the quadratic correlation function between 


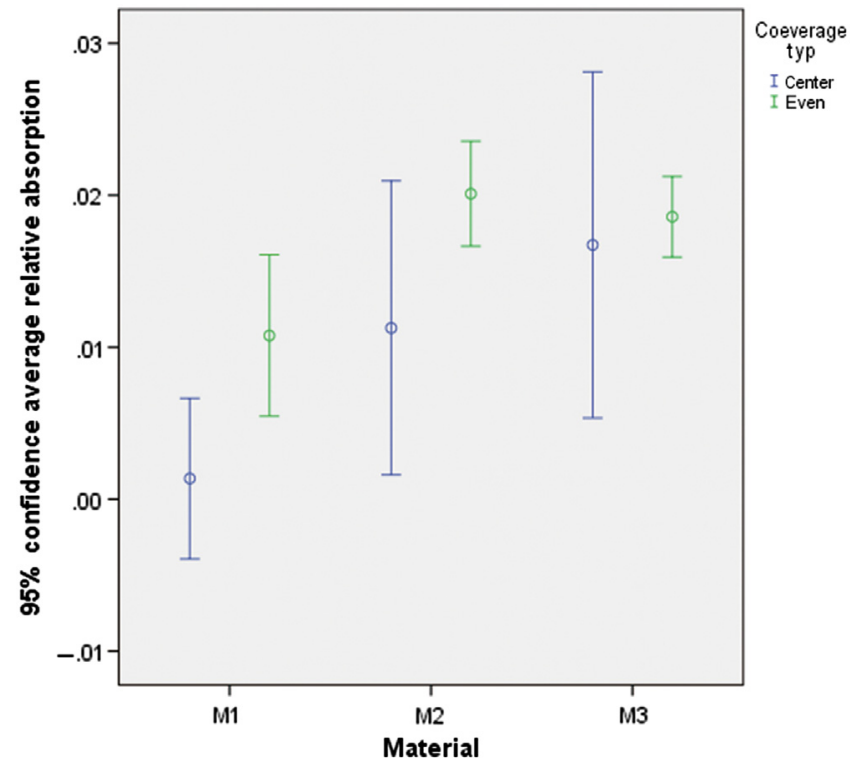

Fig. 6 Comparison of averages of the relative absorption band depths for the three materials evenly distributed as well as centered and confidence interval of the averages.

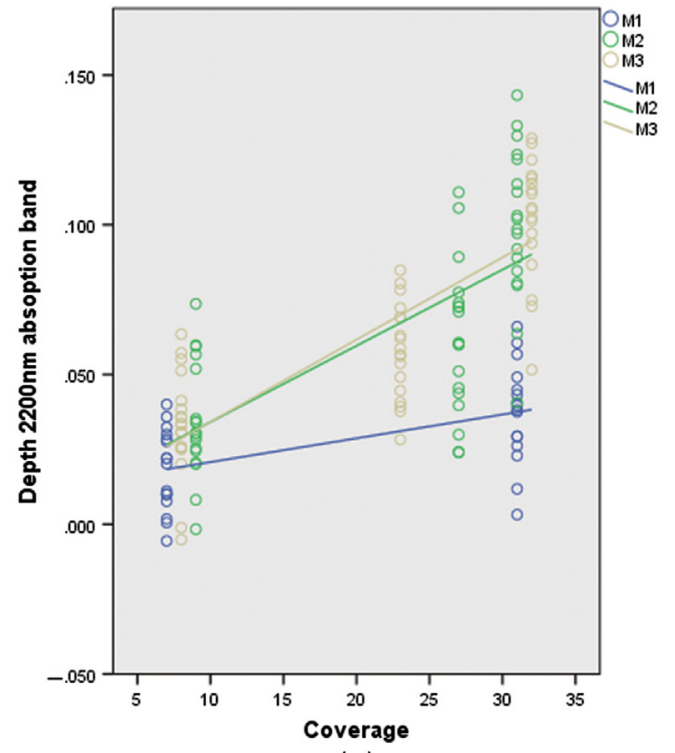

(a)

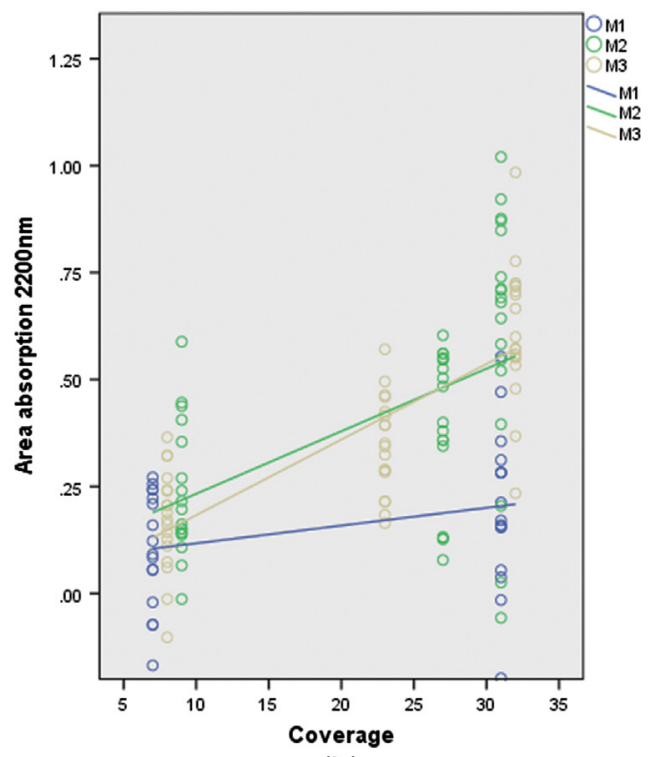

(b)

Fig. 7 (a) Depth and (b) area of the 2200-nm absorption bands summarized over all heights with a trend line.

coverage and absorption band depth. As shown in Fig. 7, this could be related to the fact that the lowest measurements were measured near the sensitivity threshold of the sensor.

\subsection{Sensitivity of the Detection in Low Coverage and Linearity in the Supporting Laboratory Experiment}

The results of the laboratory experiment were used to obtain more information on the minimum sensitivity and the linearity to expect from a calibration field. They show a high linearity between the cover of the measurement area with tailing material over the concrete and the absorption depth at $2200 \mathrm{~nm}$. The parameters of the linearity are dependent on the concrete background, but all show similar increases and an $R^{2}$ of 0.95 . Figure 8 shows the data for the four different 


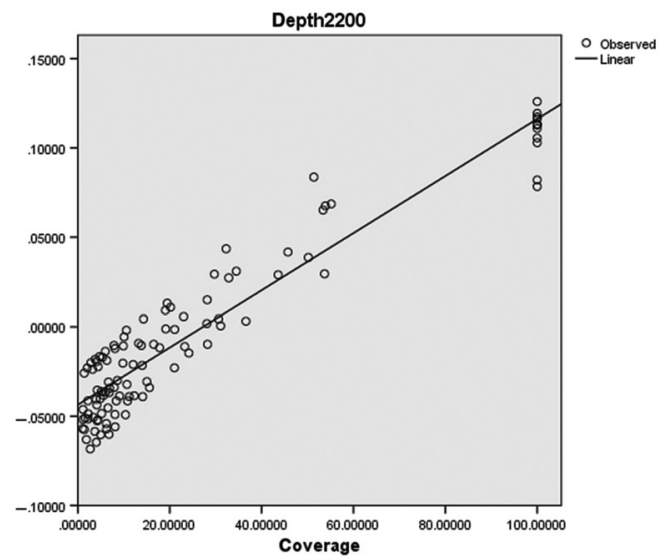

(a)

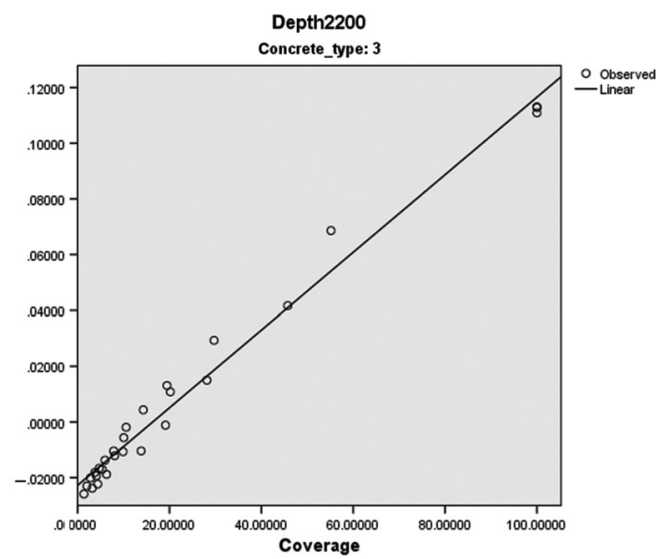

(b)

Fig. 8 Absorption band depth in normalized reflectance at $2200 \mathrm{~nm}$ of tailing material versus the coverage percent of the tailing material over the concrete in the Petri dish. (a) All concrete types together and (b) only for sample concrete type No. 3.

concrete types in the Petri dishes together-the linearity for the different concrete types is easy to see in separate point clouds. The figure also shows one example for concrete No. 3, showing the strong linearity per concrete background.

To get information on the sensitivity of the detection and the influence of the background, we analyzed the low coverage percentages of the tailings materials (low coverage exposes more background). Figure 8 shows the results for the measurements below $10 \%$ coverage. The graph shows that the linearity can be identified from coverage values between $2 \%$ and $6 \%$ coverage. In summary, the concrete types with the lowest water content showed the highest reflectance values and the best linearity between low coverage values and the absorption depth at $2200 \mathrm{~nm}$ of the tailing material. In contrast, the concrete types with higher water content showed higher variability (the extreme example is concrete type 4, Fig. 9). Concrete type No. 2 shows a good sensitivity below $4 \%$ coverage with the tailing material. Using the 1400- and 1900-nm absorption band depths as measure of the free water in the concrete indicates that concrete types 1 and 4 have the highest free water content. This affirms the importance of in-depth knowledge of the utilized background material in the field and in laboratory experimental set ups for quantitative spectral analyses

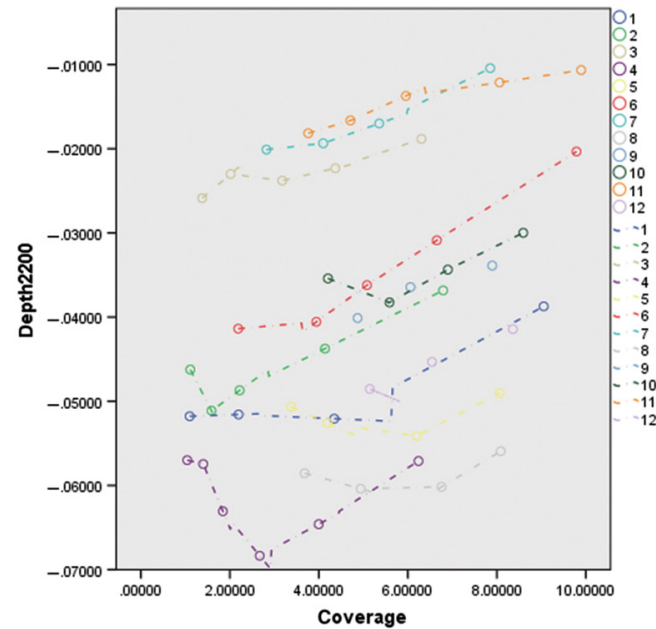

(a)

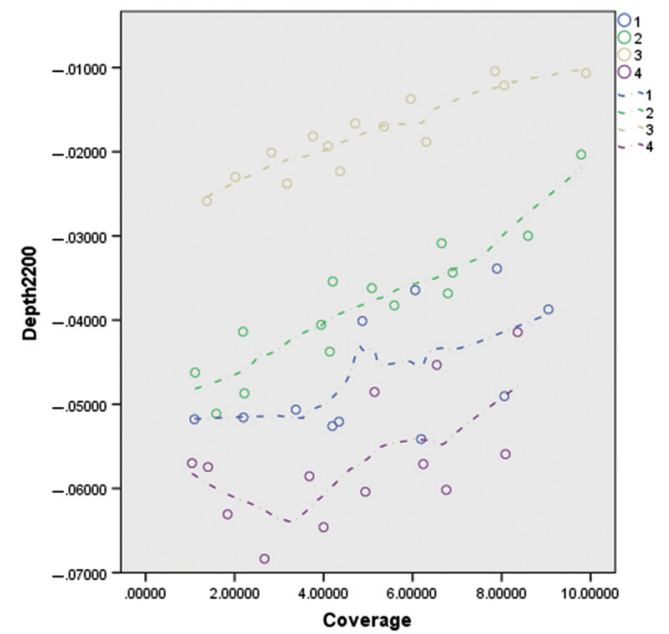

(b)

Fig. 9 (a) Measurements of relative absorption band depth $2200 \mathrm{~nm}$ on continuum-removed data in tailing material for lowest coverages in percent on the $x$ axis for the 12 sets of data and (b) summary for the four different concrete types for the same parameter. 


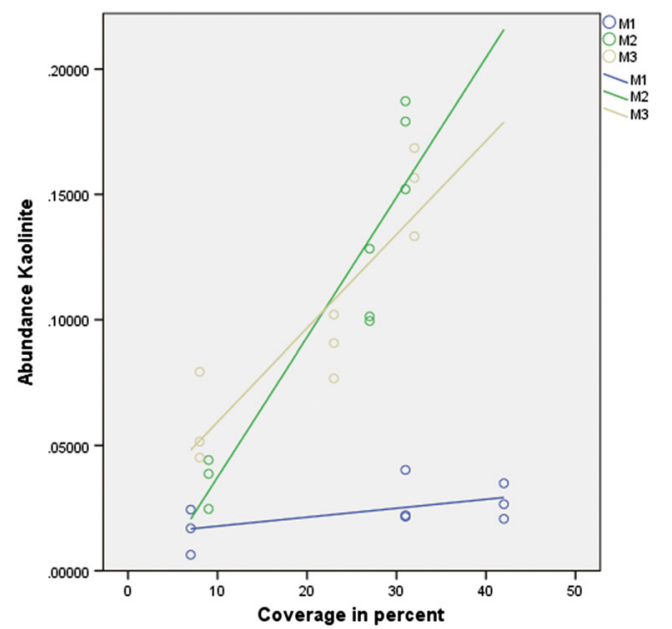

(a)

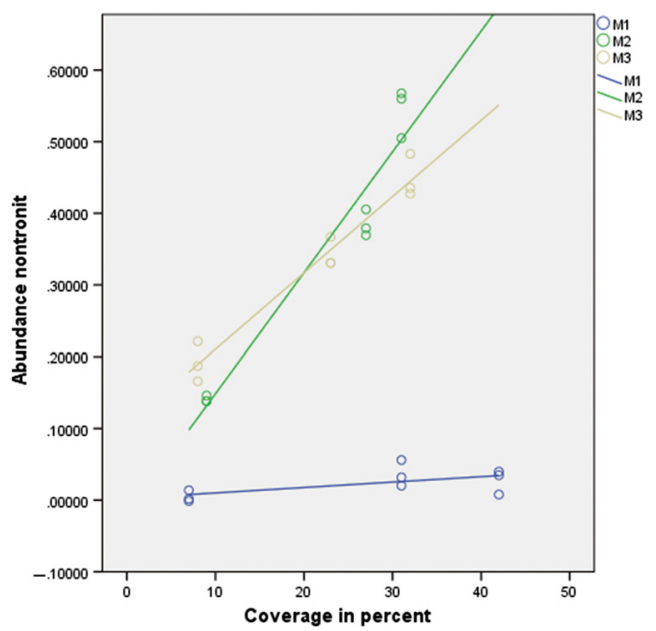

(b)

Fig. 10 Abundance of (a) kaolinite and (b) nontronite separately measured over the calibration tiles based on linear unmixing for the three target minerals.

\subsection{Separation of the Clay Minerals Using Unmixing Methods}

To separate the individual clay minerals, a linear unmixing approach was carried out using the USGS spectral library ${ }^{16}$-based spectra and the field spectra for the concrete. Then, abundance data for evenly filled calibration tiles for the materials M1 to M3 were collected. Figure 10 shows the results for the repeats for the flight altitude at $650 \mathrm{~m}$. Strong correlations between the tile material coverage and the abundance values were found for the materials M2 and M3 for the clay species nontronite $\left(R^{2}=0.93\right)$ and kaolinite $\left(R^{2}=0.87\right)$ as well as chlorite $\left(R^{2}=0.76\right)$ for material M2. For material M3, nontronite shows again the strongest correlation, while chlorite has an $R^{2}$ of 0.88 , stronger than kaolinite $\left(R^{2}=0.8\right)$. Material M1 shows a weaker correlation in the estimation of the abundance with linear unmixing, with illite with $R^{2}$ of 0.53 , and kaolinite with $R^{2}$ of 0.32 showing the strongest dependencies. As shown in Fig. 9, the increase of the abundance signal with the coverage in M1 is very small compared to the strong increase in the oxidized M2 and M3. The concrete abundance value is decreasing as expected with the increasing coverage of target material strongly independent of the cover material $\left(R^{2} \mathrm{~s}\right.$ between 0.59 for M1 and 0.95 for M3).

Comparing the results of unmixing, it shows clearly the differences in the quantity measurements using various methodologies. For instance, nontronite has strong spectral features of the SWIR and the microscopic mid-IR samples, but cannot be quantified with standard sample preparation for XRD. This points to the importance of special sample preparation of ground samples and the selection of different approaches of quantification measurements for correlation with spectral measurements. It also enforces the point that small abundances of clay minerals are important to exploration geologists and small abundances can be detected with IR. It also shows the opportunities to understand, with the help of such experiments and calibration sites, locally specific sensitivity and the relationship of individual clay minerals and apply this data to mineral exploration.

\subsection{Adaption of the Data into the Larger Area Airborne Mineral Exploration}

The calibration field was analyzed in the context of a larger survey of $160 \mathrm{~km}^{2}$, where the calibration site was utilized as a check for the whole map area. The coverage based on the calibration of the depth of the 2200-nm absorption band shows reasonable results over the mine where the rock material comes from, but also for known exploration targets. These results are based on the continuum removed data for the whole area. Applying linear unmixing to the area mapping data with similar library data as for the calibration site and using again the calibration 


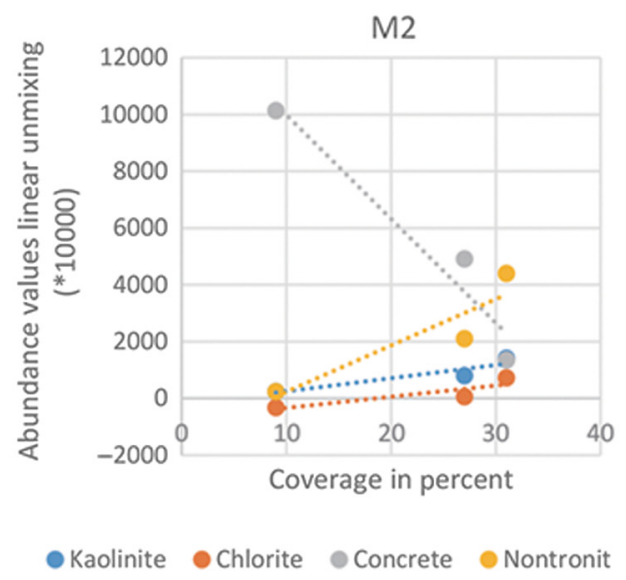

(a)

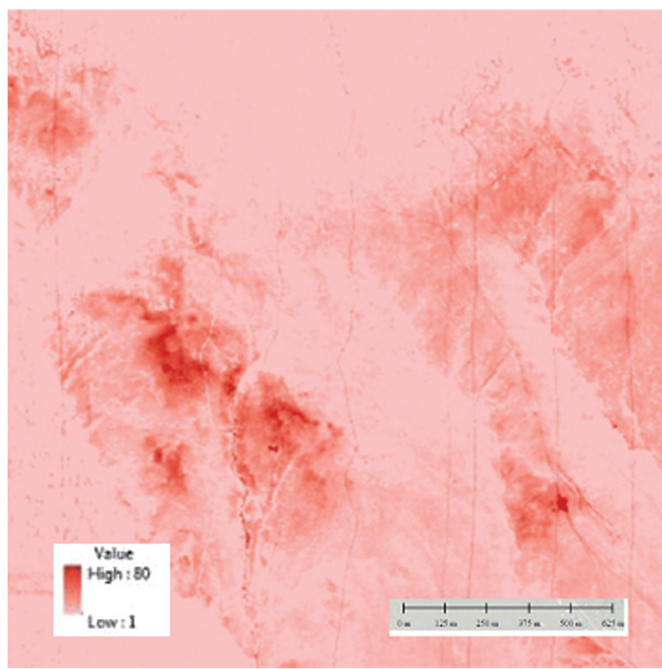

(b)

Fig. 11 (a) Results of the airborne mapping linear unmixing for some clay minerals measured over the calibration site and (b) sample map of calibrated nontronite values for a known exploration area north of Erdenet city.

site as a check, it shows similar behaviors for kaolinite, chlorite, and nontronite (Fig. 11). The example map shows the calibrated appearance based on nontronite-containing material for an area of known oxidization similar to material M2/M3. While the values using the correlation found on the calibration site are in a reasonable range, it is expected that the highest value are especially over represented and a better calibration with more different materials would improve the results.

\section{Discussion}

The calibration area was set up to be an imperfect grid in order to approximate the pixel sampling scenarios that occur in a natural setting. Therefore, resampling during rectification resulted in mixed pixels that are not real measurements, but are mathematical mixtures. This reality undoubtedly influenced the measurements, especially for higher flying altitudes with larger pixel sizes. The usage of only the center with sample material-filled tiles was only for a large measured pixel sensible due to the remaining rectification inaccuracies of the spectral airborne data. Better statistical results were achieved with evenly laid out material coverages for the whole pixel. Future calibration fields should be set up straight on the coordinate system to minimize resampling impacts and use only evenly laid out materials. The minimum size of the test tiles should be better than 1.4 times of the largest pixel size of the airborne imagery to assure the spatial accuracy of the measurements to realize the orthorectification process. Alternative inverse mapping rectification applied to the data for the calibration field could improve the spectral signature measurements. This would allow measurements on the unresampled pixels on the calibration site. As the further workflow for inverse mapping is not completely set for this study, this approach was not followed further.

The selection of the material for the calibration tiles is also of importance. The layout of the even covered material should be planned on a fine grid laid over the calibration tile. The grid should be 0.5 times the lowest airborne pixel resolution to assure even pixel coverage. For this calibration field, the discrepancy between planned and realized coverage was up to $18 \%$. The rock materials used for the calibration tiles should be as small as possible while still representing typical material of the exploration area. Using bulkier rocks, the shadow part generated by the rocks increases. This has a direct effect on the spectral data collected. For the calibration tiles with higher coverages, the shadow part was up to $14 \%$ of the tile. In any case, a high-resolution image should be used to measure the actual coverage of the calibration tiles. 
The results of the study show that the calibration field can be seen better at lower altitudes than the actual airborne exploration. This reduces the material requirements and costs for the set up of the calibration field and might allow the set up of different target rock materials.

One further effect not considered in the sensor calibration and in the results shown above is the shape of recorded data for the data cubes on the ground. For processing reasons, it is assumed that the areas have square pixels. But the recording is influenced by the design of the optical sensors (pushbroom, gating, and binning) and by the aircraft and operations condition generating the pixel (integration time, shutter time, and aircraft speed). It can be estimated that the recorded data are based on an ellipse that is mainly shaped by the aircraft speed. Multiple repeated overflights helped this study to improve the statistical reliability of the found correlations between coverage and absorption band measurements, eliminated operational shortcomings due to for instance turbulences, and should be standard in flying over calibration fields.

The atmospheric correction has a large impact on the quality of the results acquired based on a calibration field. For this study, a Modtran-based correction was used to have compatibility between the calibration field and the large area airborne survey data. Similar conditions and the close proximity of the calibration field to the exploration site should be standard. Further research on the sample data from the Erdenet calibration site will deepen the understanding of the influence of atmospheric correction and the influence of data collection on different days. In addition, the influence of the flight direction on the quantitative measurements is at this stage unclear and might have had an influence on the stability of the calibration. Only data from similar flight headings between the calibration site and airborne mission were used for this publication. The set up of clear spectral calibration targets is also important. In this study, the concrete used as background for the calibration field showed similar reflectance values to the rock material and the black net with significantly lower reflectance. In this study, this was used for the atmospheric correction, but also for the cross check of the results of the linear unmixing results with the reduction of concrete abundance with increasing coverage with target rocks. It is also germaine to discuss what background material in general to use for the calibration field. In general, concrete is not representative for geological scenes, but gives a good homogenous background. It is important to investigate what more natural background than concrete might be possible to use and how detailed this background has to be for specific exploration types. This can also be of interest in exploration surveys in remote locations where the distance between the calibration field on a concrete background and the area of interest of the exploration area might be too large or local material cannot be transported.

The main value of the calibration field is to better understand the mineralogical abundances and links to absorption strength as measured remotely. Clays are only a relatively small component of the geological target rocks used here, as is often the case in nature. Therefore, it is important to verify the identity, content, and textural occurrence of clays in the rocks to understand the abundance calibrations. The portion of the different clay minerals and the overall amount need to be measured to quantify the target lithology accordingly. This means that the rock material for the calibration field should be carefully selected and should represent the target types. Target types might be specific rock types of deposits, but can also be high-quality indicator rocks with high concentrations of the clay minerals.

The usage of the calibration field with absorption bands (in this study, mainly $2200 \mathrm{~nm}$ ) and for linear unmixing for specific clays species allows a calibrated two-way approach for quantitative measurements. The unspecific absorption band results are expected to be more robust to apply to larger areas while the calibrated linear unmixing has higher requirements on the calibration field. For a good linear unmixing calibration, the major rock types for the whole exploration area should be laid out to assure the representation in the linear unmixing of the airborne exploration data. Further studies should use both datasets and additional datasets available from modern multisensory airborne missions.

Figure 12 shows in the scatterplot a forked point cloud for the calibrated coverage based on the measured 2200-nm absorption band depth and calibrated coverage based on nontronite abundance measurement for the sample area north of Erdenet city. Marking the two upper parts of the point cloud which will be laying definitely over the minimum sensitivity of the detection of the mineral with different colors it shows that the neighboring hills have clearly distinct spectral character. It seems that on one, the nontronite-based coverage values are systematically smaller 

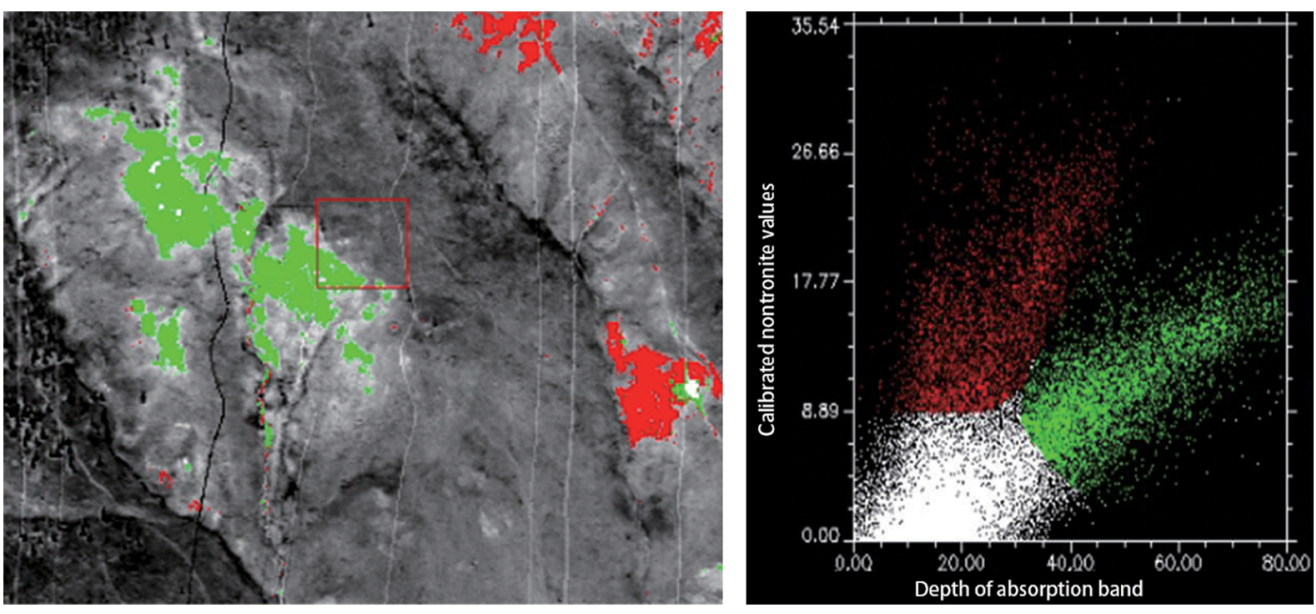

Fig. 12 Sample of spectrally mapped regional difference to calibration field-calibrated nontronite values in an exploration area north of Erdenet city.

than on the other hill. This effect cannot be found when compared to a kaolinite-based calibrated coverage. The reasons for this will have to be investigated on the ground but are expected to be based on lithological differences.

\section{Conclusion}

This paper presents the set up and the performance of a calibration field for airborne hyperspectral surveys for mineral mapping. It was developed to investigate the possibilities of quantitative estimations of target materials in the surface expression. The focus of this paper is on the methodology of the set up of the calibration field, but also presents the suitability of the approach for large-scale mineral mapping and hyperspectral hard rock scenes based on the clay absorption bands. It could be shown that the relationships between spatial resolutions, contents of the target minerals in the pixel, and measured spectral features can be visualized and can be used for modeling of quantitative measurements. The usage of a tightly controlled calibration field for specific surveys also allows localized approaches for the quantitative estimation of key mineral contents in exploration projects. The shown benefit of a calibration field-derived model in an example of the separation of regional differences in same target minerals, which will have a direct impact on the time and costs of the field exploration and drilling.

The research showed the importance of planning the calibration field in a certain direction (optimized for flight directions), tile size in relation to the expected targets to detect, and mixture of key minerals with base material, but also the selection of calibration targets and background material for the calibration site.

Further studies on the improvement of the calibration field design and the usage of other absorption features than clay bands will have to verify the usability of the methodology for other key minerals and deposit types. The data of the Erdenet calibration field will be further evaluated for artificial intelligence developments, where calibration fields can provide labels for the machine learning processes. Further, the concept of the calibration field can also be applied to small-scale target detection applications in mineral mapping such as dykes. Here, a calibration field with centered and evenly laid out targets provides, under similar to flight conditions, information on minimum sensitivity and minimum filling of a pixel to detect the target and define the parameters for the flight mission, and therefore, the economics of the exploration.

\section{Acknowledgments}

The publication is part of the project ADRIANA, sponsored by the Client II program of the German Federal Ministry of Research BMBF. ADRIANA is handled in a consortium of 
G.E.O.S. Ingenieurgesellschaft mbH, Martin-Luther-University, DIMAP-Spectral GmbH and CBM GmbH supported by the Mongolian partners Erdenet Mining (EMC), Erdenet Institut for Technology (EIT) and German-Mongolian Institute for Technology (GMIT). The consortium thanks the staff members for EMC, EIT, and GMIT for the help during the fieldwork.

\section{References}

1. R. Clark and T. Roush, "Reflectance spectroscopy: quantitative analysis techniques for remote sensing applications," J. Geophys. Res.-Solid Earth 89(B7), 6329-6340 (1984).

2. T. Cudahy, M. Caccetta, and M. Thomas, "Satellite-derived mineral mapping and monitoring of weathering, deposition and erosion," Sci. Rep. 6(23702), 1-12 (2016).

3. C. Gomez et al., "Sensitivity of clay content prediction to spectral configuration of VNIR/ SWIR imaging data, from multispectral to hyperspectral scenarios," Remote Sens. Environ. 204, 18-30 (2018).

4. J. Michalski et al., "Emission spectroscopy of clay minerals and evidence for poorly crystalline aluminosilicates on Mars from Thermal Emission Spectrometer data," J. Geophys. Res. 111, E03004 (2006).

5. G. Gilles et al., "Clay minerals mapping from imaging spectroscopy," in Earth Observation and Geospatial Analyses, pp. 1-14, IntechOpen, Rijeka (2019).

6. S. Salehi and S. Thaarup, "Mineral mapping by hyperspectral remote sensing in West Greenland using airborne, ship-based and terrestrial platforms," Geol. Survey Denmark Greenland Bull. 41, 47-50 (2018).

7. R. J. Murphy et al., "Mapping clay minerals in an open-pit mine using," Eur. J. Remote Sens. 48, 511-526 (2015).

8. F. Kruse, A. Lefkoff, and J. Dietz, "Expert system-based mineral mapping in northern Death Valley, California/Nevada, using the Airborne Visible/Infrared Imaging Spectrometer (AVIRIS)," Remote Sens. Environ. 44(2-3), 309-336 (1993).

9. D. Riley and C. A. Hecker, "Mineral mapping with airborne hyperspectral thermal infrared remote sensing at cuprite, Nevada, USA," in Thermal Infrared Remote Sensing: Sensors, Methods, Applications, Remote Sensing and Digital Image Processing, Vol. 17, pp. 495514, Springer, Dordrecht (2013).

10. L. Markelin et al., "Calibration and validation of hyperspectral imagery using a permanent test field," in 5th Workshop on Hyperspectral Image and Signal Processing: Evolution in Remote Sensing, WHISPERS 2013, Gainesville, Florida (2013).

11. D. A. Singer, V. I. Berger, and B. C. Moring, "Porphyry copper deposits of the world: database and grade and tonnage models," (Open-File Report 2008-1155), U.S. Geological Survey.

12. S. Gerel et al., "Geochemistry of granitoids and altered rocks of the Erdenet porphyry copper-molybdenum deposit, central Mongolia," in Mineral Deposit Research: Meeting the Global Challenge, pp. 1137-1140, Springer, Berlin, Heidelberg (2005).

13. A. Brook and E. Ben-Dor, "Supervised vicarious calibration of hyperspectral remote sensing data," Remote Sens. Environ. 115, 1543-1555 (2011).

14. D. Schlaepfer, M. Schaepman, and K. Itten, "PARGE: parametric geocoding based on GCP-calibrated auxiliary data," Proc. SPIE 3438, 334-344 (1998).

15. D. Schlaepfer and R. Richter, "Recent developments in ATCOR for atmospheric compensation and radiometric processing of imaging spectroscopy data," in EARSeL eProc. Vol. 14, No. 2, pp. 40-48 (2016).

16. R. F. Kokaly et al., "USGS Spectral Library Version 7,"-Data Series 1035 (2017)

Holger Eichstaedt studied Forestry at Technical University of Dresden Germany. After achieving his doctorate and further mathematical studies he established an airborne survey company which works today worldwide with focus on hyperspectral multisensory surveys.

Tugsbuyan Tsedenbaljir studied geology and economics in Ulaanbatar and is coordinating research and collaboration projects at Erdenet Institute of Technology. 
Eichstaedt et al.: Quantitative estimation of clay minerals in airborne hyperspectral data...

Rene Kahnt has a PhD in theoretical physics and leads the Department of Simulations at G.E.O.S. with focus on deposit modelling.

Michael Denk achieved his PhD at the Martin-Luther-University of Halle, Germany.

Yaron Ogen is a PhD from Tel Avic University and is also a postdoc an Martin-LutherUniversity Halle.

Cornelia Glaesser is professor emeritus at the Martin-Luther-University Halle.

Ralf Loeser is an engineer working at G.E.O.S. with focus on 3D modelling.

Rudolf Suppes studied mining at RWTH Aachen and his PhD student.

Undrakhtamir Alyeksandr studied geology and works in the exploration Department of Erdenet Mining Corporation.

Tsedendamba Oyunbuyan studied geology and works in the exploration Department of Erdenet Mining Corporation.

Joseph Michalski is a professor for remote sensing at Hong King University with focus on research around the detection of clay minerals. 